\title{
GENERALIZED MINKOWSKI FORMULAE FOR COMPACT SUBMANIFOLDS OF A RIEMANNIAN MANIFOLD
}

\author{
GEOFFREY HOWARD SMITH \\ (Received 20 July 1982, revised 23 August 1982) \\ Communicated by K. Mackenzie
}

\begin{abstract}
In $1903 \mathrm{H}$. Minkowski obtained two integral formulae for closed convex surfaces in three dimensional Euclidean space. In this paper we obtain generalised Minkowski formulae on compact orientable immersed submanifolds of an arbitrary Riemannian manifold. By successive specialisation we indicate how known integral theorems can be obtained as particular cases of our result.
\end{abstract}

1980 Mathematics subject classification (Amer. Math. Soc.): 53 C 42, 53 C 40.

\section{Introduction}

In 1903, H. Minkowski [9] obtained the following two integral formulae for a closed convex surface $M$ in Euclidean 3-space $E^{3}$ :

$$
\int_{M}(1+p H) d A=0, \quad \int_{M}(H+p K) d A=0,
$$

where $H$ and $K$ are respectively the mean curvature and Gaussian curvature of $M$ and $p$ is the function of support.

In order to generalise these equations to a submanifold of a Riemannian manifold we need a replacement for the position vector which appears via the support function $p$. One method of doing this is to assume (as was done by Katsurada [6]) that the enveloping space admits a conformal vector field. However, this is sometimes an unnecessarily restrictive assumption and it is preferable

(C) 1984 Australian Mathematical Society $0263-6115 / 84 \$ A 2.00+0.00$ 
to assume only that there is a conformal variation of the submanifold. (We explain this concept in Section 2.) The reasons why conformal variations are preferred is discussed in [11].

In Section 2 we give a brief outline of relevant results from the theory of submanifolds of a Riemannian manifold. In Section 3 we prove a sequence of integral formulae which generalise the above results and we conclude in Section 4 by indicating how known results of various authors may be obtained by specialisation of the results in Section 3.

\section{Preliminary results}

We use the notation and terminology of Kobayashi and Nomizu [7] unless otherwise stated. We assume all maps and manifolds to be $C^{\infty}$.

Let $f: M \rightarrow M^{\prime}$ be an immersion of an $m$-dimensional manifold $M$ as a submanifold of an $(m+p)$-dimensional Riemannian manifold $M^{\prime}$. We denote the metric on $M^{\prime}$ by $g$ or $\langle$,$\rangle according to convenience and we give M$ the induced metric.

A vector field over the map $f$ is a map $W: M \rightarrow T\left(M^{\prime}\right)$ such that $W(x) \in$ $T_{f(x)}\left(M^{\prime}\right)$ for all $x \in M$. Since the map $f$ is an immersion it need not be injective so that it is possible to have distinct points $x, y \in M$ with $f(x)=f(y)$ but $W(x) \neq W(y)$. Hence $W$ need not be the restriction to $f(M)$ of any vector field on $M^{\prime}$. If $x \in M$ and if $U$ is a neighbourhood of $x$ on which $f$ is injective then for any vector field $W$ over $f$ we write $W^{\perp}(x)$ (respectively $W^{t}(x)$ ) for the component of $W(x)$ perpendicular (respectively tangential) to $f(U)$ at $f(x)$.

In what follows we assume that there exist $p$ linearly independent vector fields $N_{1}, \ldots, N_{p}$ over $f$ such that $N_{i}(x)=N_{i}^{\perp}(x)$ and $\left\langle N_{i}(x), N_{j}(x)\right\rangle=\delta_{i j}$ for all $x \in M$ and $i, j=1, \ldots, p$. Such vector fields are called normal vector fields over $f$.

The evaluation of tensor quantities at a point is a purely local matter and since an immersion is locally an imbedding it simplifies matters to assume that $f$ actually is an imbedding and to identity $f(M)$ and $M$. We shall do this unless the distinction between an immersion and an imbedding becomes important. The differences will be discussed as they arise.

Let $X$ and $Y$ be vector fields on $M$ and let $N$ be a normal vector field on $M$. We have [7]

$$
D_{X}^{\prime} N=-A_{N} X+\nabla_{X} N \quad \text { (Weingarten's formula), }
$$

where $D^{\prime}$ denotes covariant differentiation on $M^{\prime}$ and $-A_{N} X$ and $\nabla_{X} N$ denote the tangential and normal components of $D_{X}^{\prime} N$ respectively. (The reader is referred to [7], Chapter 7 for the reasons behind this particular choice of notation.) 
Let $N_{1}, \ldots, N_{p}$ be an orthonormal set of normal vector fields on $M$. We write $A_{i}$ for $A_{N_{i}}$. Then we have [7]

$$
D_{X}^{\prime} Y=D_{X} Y+\sum_{i=1}^{p}\left\langle A_{i} X, Y\right\rangle N_{i} \quad \text { (Gauss' formula). }
$$

Let $W$ be a vector field over $f$. For each $x \in M$ there exist open neighbourhoods $V$ of $x$ and $U$ of $f(x)$ with $f(V) \subset U$ and a smooth vector field $\mathbf{W}$ on $U$ such that $\mathbf{W}(f(y))=W(y)$ for all $y \in V$. Then, if $L$ denotes the Lie derivative, we have

$$
\left(L_{\mathbf{w}} g\right)(X, Y)=\left\langle D_{X}^{\prime} \mathbf{W}, Y\right\rangle+\left\langle D_{Y}^{\prime} \mathbf{W}, X\right\rangle .
$$

Using equations (1) and (2) we find that

$$
\begin{aligned}
\left(L_{\mathbf{w}} g\right)(X, Y)= & \left\langle D_{X} W^{t}, Y\right\rangle+\left\langle D_{Y} W^{t}, X\right\rangle \\
& -2 \sum_{i=1}^{p}\left\langle A_{i} X, Y\right\rangle\left\langle N_{i}, W\right\rangle .
\end{aligned}
$$

The significance of this equation is that the right hand side depends only on $W$ and not the particular extension $\mathbf{W}$ of $W$. Hence we can unambiguously write $\left(L_{w} g\right)(X, Y)$ for $\left(L_{w} g\right)(X, Y)$ if $X$ and $Y$ are tangent to $M$.

A vector field $\xi$ over $f$ is a conformal variation of $f(M)$ in $M^{\prime}$ with conformal scalar $\Phi$ if $\left(L_{\xi} g\right)\left(f_{*} X, f_{*} Y\right)=2 \Phi\langle X, Y\rangle$ for some scalar $\Phi$ and any vector fields $X$ and $Y$ on $M$. This concept is preferable to assuming that the enveloping space admits a conformal vector field as is done by Katsurada [6] and subsequent authors. The reader is referred to [11] for reasons as to why this is so.

Let $e_{1}, \ldots, e_{m}$ be an orthonormal moving frame on some open set $V$ in $M$. It is possible to choose the frame so that at a point $x \in V$ the vectors $e_{1}, \ldots, e_{m}$ are eigenvectors for the self-adjoint operator $A=A_{1}$ with corresponding eigenvalues $\lambda_{1}, \ldots, \lambda_{m}$. For $k=1,2, \ldots, m$ we define

$$
\begin{aligned}
\boldsymbol{\sigma}_{k} & =\sum_{i_{1}<i_{2}<\cdots<i_{k}} \lambda_{i_{1}} \lambda_{i_{2}} \cdots \lambda_{i_{k}}, \\
\left(\begin{array}{c}
m \\
k
\end{array}\right) H_{k} & =\sigma_{k}, \quad M_{k}=\operatorname{tr}\left(A^{k}\right),
\end{aligned}
$$

while for the sake of consistency we put $H_{0}=1=\sigma_{0}, M_{0}=m$.

The following results are well-known (for example [8]):

$$
\begin{gathered}
(-1)^{r+1} r \sigma_{r}=\sum_{k=0}^{r-1}(-1)^{k} M_{r-k} \sigma_{k}, \\
\sigma_{r}=\sum_{\substack{j_{1}+2 j_{2}+\cdots+r j_{r}=r \\
j_{1} \geqslant 0}} \frac{(-1)^{j_{i}+j_{2}+\cdots+j_{r}+r} M_{1}^{j_{1}} \cdots M_{r}^{j_{r}}}{2^{j_{2}} 3^{j_{3}} \cdots r^{j_{r}} j_{1} ! \cdots j_{r} !}
\end{gathered}
$$


For any vector field $X$ on $M$ we deduce from equation (5) that

$$
X\left(\sigma_{r}\right)=\sum_{k=1}^{r}(-1)^{k+1} \frac{1}{k} \sigma_{r-k} X\left(M_{k}\right) .
$$

Let

$$
A e_{\alpha}=\sum_{\beta} \mu_{\alpha}^{\beta} e_{\beta}
$$

with

$$
\left(\mu_{\alpha}^{\beta}\right)_{x}= \begin{cases}\lambda_{\alpha} & \text { if } \alpha=\beta \\ 0 & \text { if } \alpha \neq \beta\end{cases}
$$

For any vector field $X$ on $V$ we have

$$
\begin{aligned}
X_{x}\left(M_{k}\right) & =X_{x}\left(\operatorname{tr} A^{k}\right) \\
& =X_{x}\left(\sum_{i_{j}} \mu_{i_{2}}^{i_{1}} \mu_{i_{3}}^{i_{2}} \cdots \mu_{i_{1}}^{i_{k}}\right) \\
& =\sum_{\alpha} k \lambda_{\alpha}^{k-1} X_{x}\left(\mu_{\alpha}^{\alpha}\right) .
\end{aligned}
$$

On the other hand

$$
\begin{aligned}
\sum_{\alpha}\langle & \left.\left(D_{X} A\right)\left(e_{\alpha}\right), \lambda_{\alpha}^{k-1} e_{\alpha}\right\rangle\left.\right|_{x} \\
& =\left.\sum_{\alpha, \beta}\left\langle D_{X}\left(\mu_{\alpha}^{\beta} e_{\beta}\right), \lambda_{\alpha}^{k-1} e_{\alpha}\right)\right|_{x}-\left.\sum_{\alpha}\left\langle A\left(D_{X} e_{\alpha}\right), \lambda_{\alpha}^{k-1} e_{\alpha}\right\rangle\right|_{x} \\
& =\sum_{\alpha} X_{x}\left(\mu_{\alpha}^{\alpha}\right) \lambda_{\alpha}^{k-1} .
\end{aligned}
$$

Hence

$$
\left.\sum_{\alpha}\left\langle\left(D_{X} A\right)\left(e_{\alpha}\right), \lambda_{\alpha}^{k-1} e_{\alpha}\right\rangle\right|_{x}=\frac{1}{k} X_{x}\left(M_{k}\right),
$$

a fact we shall use in the proof of Lemma 3.

\section{The general integral formulae}

As before let $f: M \rightarrow M^{\prime}$ be an immersion of an $m$-dimensional manifold $M$ in an $(m+p)$-dimensional manifold $M^{\prime}$. Suppose that $\xi$ is a conformal variation of $M$ in $M^{\prime}$, that is $\left(L_{\xi} g\right)(X, Y)=2 \Phi\langle X, Y\rangle$ for vector fields $X$ and $Y$ on $M$.

For non-negative integers $k, j_{1}, j_{2}, \ldots, j_{r}$ we define a covariant vector $\omega$ by

$$
\omega(X)=\omega_{j_{1} \cdots j_{r}}^{k}(X)=\left\langle M_{1}^{j_{1}} \cdots M_{r}^{j_{r}} A^{k} X, \xi\right\rangle .
$$


Then

$$
\left(D_{X} \omega\right)(X)=X(\omega(X))-\omega\left(D_{X} X\right)
$$

and after some simplification using equation (2) we find that

$$
\begin{aligned}
\left(D_{X} \omega\right)(X)= & \left\langle D_{X}\left(M_{1}^{j_{1}} \cdots M_{r}^{j_{r}} A^{k}\right)(X), \xi\right\rangle \\
& +\sum_{i=1}^{p}\left\langle A_{i} X, M_{1}^{j_{1}} \cdots M_{r}^{j_{r}} A^{k} X\right\rangle\left\langle N_{i}, \xi\right\rangle \\
& +M_{1}^{j_{1}} \cdots M_{r}^{j_{r}}\left\langle A^{k} X, D_{X}^{\prime} \xi\right\rangle
\end{aligned}
$$

We put $X=e_{\alpha}, \eta=\eta_{j_{1} \ldots j_{r}}^{k}=\hat{g}(\omega, \cdot)$ (where $\hat{g}$ is the contravariant metric tensor) and sum over $\alpha=1,2, \ldots, m$.

The result is

$$
\begin{aligned}
\operatorname{div} \eta= & \sum_{\alpha=1}^{m}\left[\left\langle D\left(M_{1}^{j_{1}} \cdots M_{r}^{j_{r}} A^{k}\right)\left(e_{\alpha}, e_{\alpha}\right), \xi\right\rangle\right. \\
& \left.\quad+\sum_{i=1}^{p} \lambda_{\alpha}^{k} M_{1}^{j_{1}} \cdots M_{r}^{j_{r}}\left\langle A_{i} e_{\alpha}, e_{\alpha}\right\rangle\left\langle N_{i}, \xi\right\rangle\right] \\
& +M_{1}^{j_{1}} \cdots M_{r}^{j_{r} \Phi} M_{k} .
\end{aligned}
$$

Now form the sum

$$
\operatorname{div} \bar{\eta} \stackrel{\text { def }}{=} \sum_{k=0}^{r<m} \sum_{j_{1}+2 j_{2}+\cdots+r j_{r}=r-k} \frac{(-1)^{j_{1}+j_{2}+\cdots+j_{r}+r} \operatorname{div} \eta_{j_{1} \cdots j_{r}}^{k}}{2^{j_{2}} \cdots r^{j_{r}} j_{1}^{!} \cdots j_{r}}
$$

We note that as $k$ assumes the values $1,2,3, \ldots$ we must have $j_{r}, j_{r-1}, j_{r-2}, \ldots$ equal to zero. Bearing this in mind and using equations (4) and (5) we find after some simplification that

$$
\begin{aligned}
\operatorname{div} \bar{\eta}= & (m-r) \sigma_{r} \Phi \\
+ & +\sum_{\alpha=1}^{m} \sum_{k=0}^{r}(-1)^{k}\left[\sum_{i=1}^{p} \sigma_{r-k} \lambda_{\alpha}^{k}\left\langle A_{i} e_{\alpha}, e_{\alpha}\right\rangle\left\langle N_{i}, \xi\right\rangle\right. \\
& \left.+\left\langle D\left(\sigma_{r-k} A^{k}\right)\left(e_{\alpha}, e_{\alpha}\right), \xi\right\rangle\right]
\end{aligned}
$$


that is

(8)

$$
\begin{aligned}
\operatorname{div} \bar{\eta}= & (m-r) \sigma_{r} \Phi+(r+1) \sigma_{r+1}\langle N, \xi\rangle \\
& +\sum_{\alpha=1}^{m} \sum_{k=0}^{r}(-1)^{k}\left[\sum_{i=2}^{p} \sigma_{r-k} \lambda_{\alpha}^{k}\left\langle A_{i} e_{\alpha}, e_{\alpha}\right\rangle\left\langle N_{i}, \xi\right\rangle\right. \\
& \left.+\left\langle D\left(\sigma_{r-k} A^{k}\right)\left(e_{\alpha}, e_{\alpha}\right), \xi\right\rangle\right],
\end{aligned}
$$

where $N=N_{1}$.

We shall obtain an alternative expression for div $\bar{\eta}$ after proving some lemmas.

\section{LEMMA 1.}

$$
\sum_{k=0}^{r}(-1)^{k} \sigma_{r-k} \lambda_{\alpha}^{k}=\sum_{\substack{i_{1}<\cdots<i_{r} \\ i, \neq \alpha \\ j=1,2, \ldots, r}} \lambda_{i_{1}} \cdots \lambda_{i_{r}}
$$

Proof. (By induction on $r$.) For $r=1$ we have

$$
\sum_{k=0}^{1}(-1)^{k} \sigma_{1-k} \lambda_{\alpha}^{k}=\sigma_{1}-\lambda_{\alpha}=\sum_{k \neq \alpha} \lambda_{k} .
$$

Assume the result to be true for $r=s$, that is assume

$$
\sum_{k=1}^{s+1}(-1)^{k+1} \sigma_{s+1-k} \lambda_{\alpha}^{k-1}=\sum_{\substack{i_{1}<\cdots<i_{s} \\ i_{j} \neq \alpha}} \lambda_{i_{1}} \cdots \lambda_{i_{s}} .
$$

Then for $r=s+1$ we have

$$
\begin{aligned}
& \sum_{k=0}^{s+1}(-1)^{k} \sigma_{s+1-k} \lambda_{\alpha}^{k}=\sigma_{s+1}-\left(\sum_{k=1}^{s+1}(-1)^{k+1} \sigma_{s+k-1} \lambda_{\alpha}^{k-1}\right) \lambda_{\alpha} \\
& =\sigma_{s+1}-\lambda_{\alpha}\left(\sum_{\substack{i_{1}<\cdots<i_{s} \\
i_{j} \neq \alpha}} \lambda_{i_{1}} \cdots \lambda_{i_{s}}\right) \\
& =\left(\sum_{i_{1}<\cdots<i_{s+1}} \lambda_{i_{1}} \cdots \lambda_{i_{s+1}}\right)-\lambda_{\alpha} \sum_{\substack{i_{1}<\cdots<i_{s} \\
i_{j} \neq \alpha}} \lambda_{i_{1}} \cdots \lambda_{i_{s}} \\
& =\sum_{\substack{i_{1}<\cdots i_{s+1} \\
i_{j} \neq \alpha}} \lambda_{i_{1}} \cdots \lambda_{i_{s+1}}
\end{aligned}
$$

The result follows. 
As an immediate consequence of this result we have

\section{LEMMA 2.}

$$
\sum_{\alpha=1}^{m} \sum_{k=0}^{r} \sum_{i=2}^{p}(-1)^{k} \sigma_{r-k} \lambda_{\alpha}^{k}\left\langle A_{i} e_{\alpha}, e_{\alpha}\right\rangle\left\langle N_{i}, \xi\right\rangle=\frac{m !}{r !(m-r-1) !} \sum_{i=2}^{p} F_{i}^{r}(N),
$$

where

$$
\text { (9) } F_{i}^{r}(N)=\frac{r !(m-r-1) !}{m !}\left\langle N_{i}, \xi\right\rangle \sum_{\substack { \alpha=1 \\
\begin{subarray}{c}{i_{1}<\ldots<i_{r} \\
i_{j} \neq \alpha \\
j=1, \ldots, r{ \alpha = 1 \\
\begin{subarray} { c } { i _ { 1 } < \ldots < i _ { r } \\
i _ { j } \neq \alpha \\
j = 1 , \ldots , r } }\end{subarray}}^{m} \lambda_{i_{1}} \cdots \lambda_{i_{r}}\left\langle A_{i} e_{\alpha}, e_{\alpha}\right\rangle \text {. }
$$

\section{LEMMA 3.}

$$
\sum_{\alpha=1}^{m} \sum_{k=0}^{r}(-1)^{k}\left\langle D\left(\sigma_{r-k} A^{k}\right)\left(e_{\alpha}, e_{\alpha}\right), \xi\right\rangle=\frac{m !}{r !(m-r-1) !}\left[S^{r}(\xi)+E^{r}(\xi)\right],
$$

where

$$
\begin{aligned}
S^{r}(\xi)= & \frac{r !(m-r-1) !}{m !} \\
& \times \sum_{\alpha=1}^{m} \sum_{s=1}^{r} \sum_{k=0}^{s-1}(-1)^{s} \sigma_{r-s} \lambda_{\alpha}^{k}\left\langle R^{\prime}\left(e_{\alpha}, A^{s-k-1} \xi^{t}\right) e_{\alpha}, N\right\rangle
\end{aligned}
$$

and

(11)

$$
\begin{aligned}
E^{r}(\xi)= & \frac{r !(m-r-1) !}{m !} \\
& \times \sum_{\alpha=1}^{m} \sum_{s=1}^{r} \sum_{k=0}^{s-1} \sum_{i=1}^{p}(-1)^{s} \sigma_{r-s} \lambda_{\alpha}^{k}\left\{\left\langle A_{i} e_{\alpha}, e_{\alpha}\right\rangle\left\langle\left(\nabla N_{i}\right)\left(A^{s-k-1} \xi^{t}\right), N\right\rangle\right. \\
& \left.-\left\langle A_{i} e_{\alpha}, A^{s-k-1} \xi^{t}\right\rangle\left\langle\left(\nabla N_{i}\right)\left(e_{\alpha}\right), N\right\rangle\right\} .
\end{aligned}
$$

The factorials are introduced for later convenience.

Proof. The proof is essentially a coordinate-free generalised version of the proof of Rund [10] for a hypersurface. We begin by noting the following two results

$$
\left\langle\left(D_{Y} A^{k}\right)(Z), X\right\rangle=\left\langle Z,\left(D_{Y} A^{k}\right)(X)\right\rangle
$$

and

$$
\left\langle\left(D A^{r}\right)\left(e_{\alpha}, e_{\alpha}\right), \xi\right\rangle=\sum_{s=0}^{r-1} \lambda_{\alpha}^{r-s-1}\left\langle(D A)\left(e_{\alpha}, e_{\alpha}\right), A^{s} \xi^{t}\right\rangle
$$


The first result follows from a straightforward calculation using the fact that $A$ is self-adjoint and the second may be proved by induction on $r$ and use of the first result.

If we put $\xi^{t}=\eta$ and

$$
E_{\alpha}(\eta)=\sum_{i=1}^{p}\left\langle\left\langle A_{i} e_{\alpha}, \eta\right\rangle\left(\nabla N_{i}\right)\left(e_{\alpha}\right)-\left\langle A_{i} e_{\alpha}, e_{\alpha}\right\rangle\left(\nabla N_{i}\right)(\eta), N\right\rangle
$$

then by Codazzi's equation [7, Volume 2] we have

(13) $\left\langle R^{\prime}\left(e_{\alpha}, \eta\right) e_{\alpha}, N\right\rangle=\left\langle(D A)\left(e_{\alpha}, e_{\alpha}\right), \eta\right\rangle-\left\langle\left(D_{\eta} A\right)\left(e_{\alpha}\right), e_{\alpha}\right\rangle+E_{\alpha}(\eta)$.

We multiply equation (13) by $\lambda_{\alpha}^{r-1}$, sum over $\alpha$, make use of equation (7), and rearrange the result to get

$$
\begin{aligned}
& \sum_{\alpha=1}^{m} \lambda_{\alpha}^{r-1}\left\langle(D A)\left(e_{\alpha}, e_{\alpha}\right), \eta\right\rangle \\
& \quad=\frac{1}{r} \eta\left(M_{r}\right)+\sum_{\alpha} \lambda_{\alpha}^{r-1}\left[\left\langle R^{\prime}\left(e_{\alpha}, \eta\right) e_{\alpha}, N\right\rangle-E_{\alpha}(\eta)\right] .
\end{aligned}
$$

Using equations (12) and (14) we have

$$
\begin{aligned}
\sum_{\alpha=1}^{m}\left\langle\left(D A^{r}\right)\left(e_{\alpha}, e_{\alpha}\right), \xi\right\rangle \\
\quad=\sum_{s=0}^{r-1}\left\{\frac{1}{r-s}\left(A^{s} \eta\right)\left(M_{r-s}\right)+\sum_{\alpha} \lambda_{\alpha}^{r-1}\left[\left\langle R^{\prime}\left(e_{\alpha}, A^{s} \eta\right) e_{\alpha}, N\right\rangle-E_{\alpha}\left(A^{s} \eta\right)\right]\right\} .
\end{aligned}
$$

We also have

$$
\sum_{\alpha} \sum_{k}(-1)^{k}\left\langle\left(D \sigma_{r-k} A^{k}\right)\left(e_{\alpha}, e_{\alpha}\right), \eta\right\rangle
$$

$$
=\sum_{k=0}^{r}(-1)^{k}\left[\left(A^{k} \eta\right)\left(\sigma_{r-k}\right)+\sum_{\alpha} \sigma_{r-k}\left\langle D A^{k}\left(e_{\alpha}, e_{\alpha}\right), \eta\right\rangle\right] .
$$

The first term on the right hand side of equation (16) may be rewritten as

$$
\sum_{k=0}^{r}(-1)^{k}\left(A^{k} \eta\right)\left(\sigma_{r-k}\right)=\sum_{s=1}^{r}(-1)^{s+r}\left(A^{r-s} \eta\right)\left(\sigma_{s}\right),
$$

which with the aid of equation (7) becomes

$$
\sum_{k=0}^{r}(-1)^{k}\left(A^{k} \eta\right)\left(\sigma_{r-k}\right)=\sum_{k=1}^{r} \sum_{s=1}^{k}(-1)^{k+r+s+1} \frac{1}{s} \sigma_{k-s}\left(A^{r-k} \eta\right)\left(M_{s}\right) \text {. }
$$


Since $D A^{0}=D I=0$ the second term on the right hand side of equation (16) becomes, on making use of equation (15)

$$
\begin{aligned}
& \sum_{k=1}^{r} \sum_{\alpha=1}^{m}(-1)^{k} \sigma_{r-k}\left\langle D A^{k}\left(e_{\alpha}, e_{\alpha}\right), \eta\right\rangle \\
& =\sum_{s=1}^{r}(-1)^{s} \sigma_{r-s}\left\{\sum _ { i = 0 } ^ { s - 1 } \left(\frac{1}{s-i}\left(A^{i} \eta\right)\left(M_{s-i}\right)\right.\right. \\
& \left.\left.\quad+\sum_{\alpha} \lambda_{\alpha}^{s-i-1}\left[\left\langle R^{\prime}\left(e_{\alpha}, A^{i} \eta\right) e_{\alpha}, N\right\rangle-E_{\alpha}\left(A^{i} \eta\right)\right]\right)\right\}
\end{aligned}
$$

The first term on the right hand side of equation (18) is seen, after some rearrangement, to be the additive inverse of the term on the right hand side of equation (17). Substitution of equations (17) and (18) into equation (16) yields the desired result.

THEOREM. Let $f: M \rightarrow M^{\prime}$ be an immersion of a compact orientable m-dimensional manifold $M$ in an $(m+p)$-dimensional Riemannian manifold $M^{\prime}$. Let $\xi$ be a conformal variation of $f(M)$ in $M^{\prime}$ with conformal scalar $\Phi$. Assume that there exists an orthonormal set $N_{1}, \ldots, N_{p}$ of normal vector fields over $f$. Let $N=N_{1}$ be one such vector field and let $A$ be the corresponding second fundamental tensor. Then for $r=0,1,2, \ldots, m-1$

$$
\begin{aligned}
\int_{M}\left(\Phi H_{r}+H_{r+1}\langle N, \xi\rangle\right) d A \\
=-\int_{M}\left\{S^{r}(\xi)+E^{r}(\xi)+\sum_{i=2}^{p} F_{i}^{r}(N)\right\} d A .
\end{aligned}
$$

where $F_{i}^{r}(N), S^{r}(\xi)$ and $E^{r}(\xi)$ are given by equations (9), (10) and (11) respectively for $r \geqslant 1$ and $S^{0}(\xi)=0=E^{0}(\xi), F_{i}^{0}(N)=\left\langle A_{i} e_{\alpha}, e_{\alpha}\right\rangle\left\langle N_{i}, \xi\right\rangle$.

Proof. By Stokes' theorem $\int_{M} \operatorname{div} \bar{\eta} d A=0$, so that for $r=1,2, \ldots, m-1$ the theorem follows on using equation (8), Lemma 2 and Lemma 3. We remark that if $f$ is an immersion then by abuse of notation the vector field $\bar{\eta}$ in equation (8) really denotes the unique field $Z$ on $M$ such that $f_{*} Z=\bar{\eta}$. It is this vector field $Z$ that Stokes' theorem should be applied.

The case $r=0$ follows from equation (3) by putting $X=e_{\alpha}=Y, W=\xi$, summing over $\alpha=1, \ldots, m$ and then applying Stokes' theorem. 


\section{Special cases of the integral formulae}

1. Let $M$ be a hypersurface of $M^{\prime}$. Then $E^{r}(\xi)=0$ and $F_{i}^{r}(N)=0$ for $i=2, \ldots, p$. Formulae (19) then reduce to

$$
\int_{M}\left(\Phi H_{r}+H_{r+1}\langle N, \xi\rangle\right) d A=-\int_{M} S^{r}(\xi) d A,
$$

a result of Rund [10]. (Note that equation (3.13) of [10] is missing a factor $(-1)^{s}$. Inserting this factor in the result (6.6) of [10] yields agreement with our equation (20).)

2. Let $M^{\prime}$ be a space of constant curvature and let $N$ be parallel in the normal bundle over $M$. Then $S^{r}(\xi)=0=E^{r}(\xi)$, so that

$$
\int_{M}\left(\Phi H_{r}+H_{r+1}\langle N, \xi\rangle\right) d A=-\int_{M_{i=2}}^{p} F_{i}^{r}(N) d A .
$$

3. In addition to the hypotheses 2 above we assume that $\xi$ is coplanar with $N$ and $N_{2}$ then equation (21) becomes

$$
\int_{M}\left(\Phi H_{r}+H_{r+1}\langle N, \xi\rangle\right) d A=-\int_{M} F_{2}^{r}(N) d A,
$$

a result of Hsiung, Liu and Mittra [5] who discuss some further special cases of this result. If $M^{\prime}$ is a Euclidean space and $\xi$ is the position vector field then equation (22) is due to Chen and Yano [2]. Chen [1, page 197] discusses several examples where $F_{2}^{r}(N)$ vanishes if the imbedding space is Euclidean.

4. If we assume $M$ to be a hypersurface of a space of constant curvature then equations (20) and (22) yield the result

$$
\int_{M}\left(\Phi H_{r}+H_{r+1}\langle N, \xi\rangle\right) d A=0
$$

(Hsiung [3], [4], Katsurada [6]).

5. Let $M^{\prime}$ be an Einstein space. Then $S^{1}(\xi)=0$. If in addition $M$ is a hypersurface of $M^{\prime}$ then we have from equation (20) with $r=0,1$

$$
\begin{aligned}
\int_{M}\left(\Phi+H_{1}\langle N, \xi\rangle\right) d A & =0, \\
\int_{M}\left(\Phi H_{1}+H_{2}\langle N, \xi\rangle\right) d A & =0 .
\end{aligned}
$$

\section{References}

[1] B. Y. Chen, Geometry of submanifolds (Marcel Dekker Inc., 1973).

[2] B. Y. Chen and K. Yano, 'Integral formulas for submanifolds and their applications,' $J$. Differential Geometry 5 (1971), 467-477. 
[3] C. C. Hsiung, 'Some integral formulas for closed hypersurfaces,' Math. Scand. 2 (1954), 286-294.

[4] C. C. Hsiung, 'Some integral formulas for closed hypersurfaces in Riemann space,' Pacific J. Math. 6 (1956), 291-299.

[5] C. C. Hsiung, J. Liu and S. Mittra, 'Integral formulas for closed submanifolds of a Riemannian manifold,' J. Differential Geometry 12 (1977), 133-151.

[6] Y. Katsurada, 'Generalised Minkowski formula for closed hypersurfaces in Riemann space,' Ann. Mat. Pura Appl. (4) 57 (1962), 283-294.

[7] S. Kobayashi and K. Nomizu, Foundations of differential geometry (Interscience Publishers 1963, 1969 (2 volumes)).

[8] P. A. MacMahon, Combinatory analysis (Chelsea Publishing Company, 1960).

[9] H. Minkowski, 'Volumen und Oberfläche,' Math. Ann. 57 (1903), 447-495.

[10] H. Rund, 'Integral formulae on hypersurfaces in Riemannian manifolds,' Ann. Mat. Pura. Appl. (4) 88 (1971), 92-122.

[11] G. H. Smith, 'Conformal variation of a hypersurface of constant mean curvature in an Einstein space,' J. A ustral. Math. Soc. Ser. A 33 (1982), 229-234.

The School of Mathematical Sciences

The N.S.W. Institute of Technology

P.O. Box 123 '

Broadway, N.S.W. 2007

Australia 